\title{
Sliding friction between two silicon-carbide surfaces
}

Cite as: J. Appl. Phys. 125, 124301 (2019); https://doi.org/10.1063/1.5086185

Submitted: 18 December 2018 . Accepted: 08 March 2019. Published Online: 26 March 2019

Nariman Piroozan, Saber Naserifar (D), and Muhammad Sahimi (D)
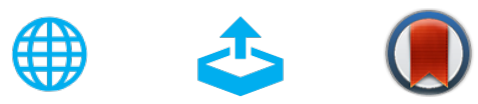

View Online

Export Citation

CrossMark

\section{ARTICLES YOU MAY BE INTERESTED IN}

Reverse breakdown studies of GaN MOSCAPs and their implications in vertical GaN power devices

Journal of Applied Physics 125, 124101 (2019); https://doi.org/10.1063/1.5082652

A perspective on topological nanophotonics: Current status and future challenges

Journal of Applied Physics 125, 120901 (2019); https://doi.org/10.1063/1.5086433

Identification of dislocation characteristics in Na-flux-grown GaN substrates using bright-field $\mathrm{X}$-ray topography under multiple-diffraction conditions

Journal of Applied Physics 125, 125105 (2019); https://doi.org/10.1063/1.5082990

\section{Applied Physics Reviews} Now accepting original research

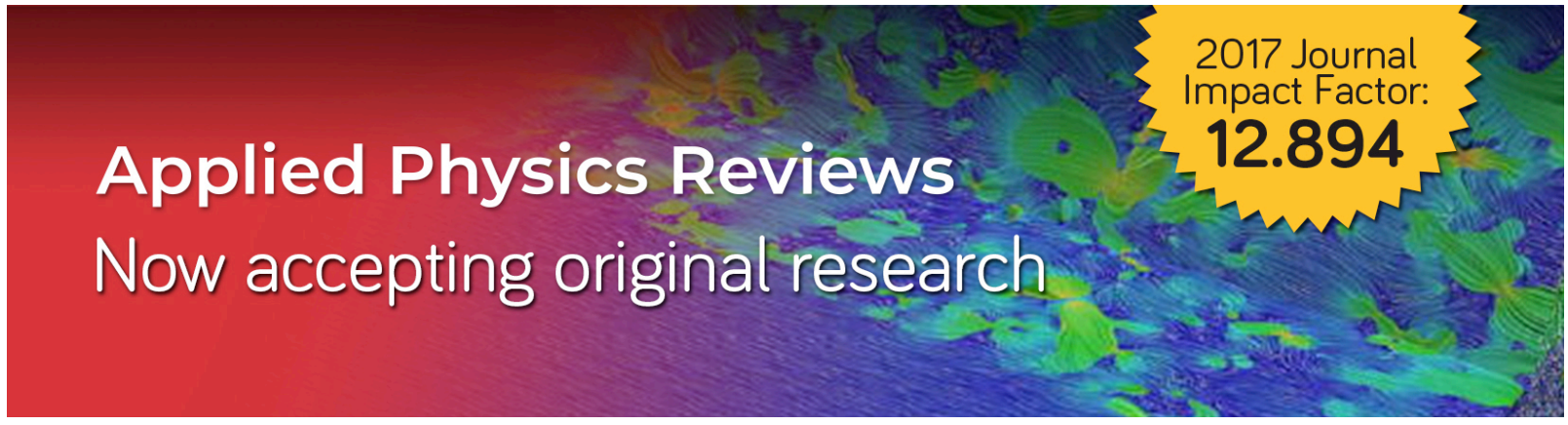




\title{
Sliding friction between two silicon-carbide surfaces
}

\author{
Cite as: J. Appl. Phys. 125, 124301 (2019); doi: 10.1063/1.5086185 \\ Submitted: 18 December 2018 . Accepted: 8 March 2019. \\ Published Online: 26 March 2019
}

Nariman Piroozan, ${ }^{7}$ Saber Naserifar, ${ }^{2}$ (D) and Muhammad Sahimi ${ }^{1, a)}$ (iD

\begin{abstract}
AFFILIATIONS
${ }^{1}$ Mork Family Department of Chemical Engineering and Materials Science, University of Southern California, Los Angeles, California 90089-1211, USA

${ }^{2}$ Materials and Process Simulation Center, California Institute of Technology, Pasadena, California 91125, USA
\end{abstract}

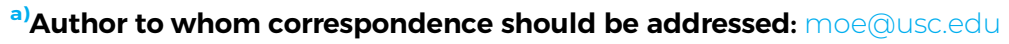

\begin{abstract}
Sliding friction between two $\mathrm{SiC}$ surfaces is important due to its relevance to many practical applications. It is also important to study whether kinetic friction at the nanoscale follows Coulomb's law. Since SiC exists both as an amorphous material and with a crystalline structure, the effect of surface roughness on the kinetic friction may also be significant. We report the results of an extensive molecular dynamics simulation of sliding friction between surfaces of the two types of $\mathrm{SiC}$ over a wide range of sliding velocities. The amorphous $\mathrm{SiC}$ was generated by the reactive force field ReaxFF, which was also used to represent the interaction potential for the simulation of sliding friction. As the sliding velocity increases, bond breaking occurs at the interface between the two surfaces, leading to their roughening and formation of excess free volume. They reduce the kinetic friction force, hence resulting in decreasing the difference between kinetic friction in the amorphous and crystalline surfaces. The average kinetic friction force depends nonlinearly on the sliding velocity $V$, implying that Coulomb's law of friction is not satisfied by the surfaces that we study at the nanoscale. The average kinetic friction force $F_{k}$ depends on $V$ as $F_{k} \propto \ln V$.
\end{abstract}

Published under license by AIP Publishing. https://doi.org/10.1063/1.5086185

\section{INTRODUCTION}

Sliding or kinetic friction between two surfaces in contact, when one of them is moving, has been a fundamental scientific problem since several centuries ago ${ }^{1}$ and, due to its ubiquity in all types of systems, ${ }^{2}$ has gained even more importance in the modern era. The direction of kinetic friction is opposite to that of the relative motion of the two surfaces in contact, but it need not oppose the net external force between them.

One type of kinetic friction is between dry surfaces, which is usually described by Coulomb's law ${ }^{3}$ according to which the magnitude of the kinetic friction force $F_{k}$ is given by $F_{k}=\mu F_{n}$, where $\mu$ is the coefficient of kinetic friction and $F_{n}$ is the normal force between the two surfaces in contact. Thus, according to Coulomb's law, the magnitude of the kinetic friction exerted through the surface is independent of the magnitude of the sliding velocity $V$ of the surfaces against each other. Apart from Coulomb's law, however, there are many other laws that link the static (contact pressure) and kinematic (velocity and hold time) quantities with the frictional force or tangential frictional tractions. Examples include the so-called Shaw's law for saturation of frictional force ${ }^{4}$ and the rate-and-state family of friction laws. ${ }^{5}$
Wet or lubricated sliding friction is the second type of phenomenon. In this case, a fundamental concept is the so-called Stribeck curve, ${ }^{6,7}$ which demonstrates that friction in fluid-lubricated contacts is a nonlinear function of the contact load, the lubricant's viscosity, and its entrainment speed. Nikolai Pavlovich Petrov was presumably the first ${ }^{2}$ who studied the effect of lubricants upon friction of journal bearings. Petrov recognized that understanding of friction between sliding lubricated surfaces is obtained by studying it as a problem in hydrodynamics and derived a simple equation, often referred to as Petrov's law, for the kinetic friction force on a journal bearing. In fact, Petrov's law represents the linear part of the Stribeck curve and holds for high values of the lubrication parameter $L$, i.e., $L=$ viscosity $\times$ velocity/pressure. More generally, Stokes's equation is used to model lubricated contacting surfaces, with the result representing an average over the thickness and usually referred to as Reynolds's equation. According to Reynolds's equation, $F_{k} \propto V \eta / D$, where $\eta$ is the viscosity and $D$ is the thickness of the lubricating film. But, in general, wet sliding friction is a complex phenomenon, due to the fact that at low velocities/high pressure, lubrication is mixed with intimate contact that increases 
friction considerably, and at very low velocities a pure boundary lubrication holds. But, neither Coulomb's law nor any other law that governs wet sliding friction takes into account, at least explicitly, the effect of the chemical structure of the two contacting surfaces, as well as the morphology of the interfacial area.

While lubricated sliding friction is relatively well understood, and the validity of Reynolds's law has been demonstrated ${ }^{8,9}$ over a range of length scales, the work on dry friction is still continuing because the phenomenon of stick-slip is widespread and occurs over widely disparate length scales, ranging from fault motion in rock that gives rise to seismic events and earthquakes, ${ }^{10,11}$ serrated yielding in metals, ${ }^{12}$ and curve squeal in rail wheels, ${ }^{13}$ to various phenomena at the atomistic scale, such as abrasive wear on aluminum alloy substrates, ${ }^{14}$ friction of krypton monolayers sliding on a gold surface, ${ }^{15}$ friction between silicon (Si) tips in a friction-force microscope and various carbon compounds, ${ }^{16}$ as well as on a $\mathrm{NaCl}$ (100) surface $^{17}$ and unzipping of DNA strands. ${ }^{18}$ The most important finding of the research on sliding friction in some materials at the atomic scale has been that, at such scales, it does not necessarily follow Coulomb's law. For example, in the aforementioned experiments on the $\mathrm{NaCl}(100)$ surface, it was found that ${ }^{18}$ the friction force at low sliding velocities $V$ varies as $F_{k} \propto \ln V$, which is a quite weak dependence on the sliding velocity.

Since Coulomb's law may be violated at the nanoscale, understanding the origin of the violation requires molecular dynamics (MD) simulation. Such simulations make it also possible to include the precise chemical composition and structure of the sliding surfaces and study their effect. Several significant efforts have been made in this direction ${ }^{19-32}$ for studying sliding friction between various surfaces. The goal of the present paper is to report on the results of our study of the phenomenon between two siliconcarbide $(\mathrm{SiC})$ surfaces, using MD simulations.

Due to its many unique properties, such as high thermal conductivity, ${ }^{33}$ thermal shock resistance, ${ }^{34}$ biocompatibility, ${ }^{35}$ resistance to acidic and alkali environments, ${ }^{36}$ chemical inertness, and high mechanical strength, ${ }^{37,38} \mathrm{SiC}$ is a highly important material. In addition to its use in the electronic and computer industries, $\mathrm{SiC}$ has also been used for fabricating nanoporous membranes ${ }^{39,40}$ and nanotubes. ${ }^{41-43}$ But what has motivated us to study sliding friction between two $\mathrm{SiC}$ surfaces is its applications in high performance disc brakes, cutting tools, and blades in gas turbines and jet engines, as well as fabrication of nanoporous materials explained below. Consider, for example, the development of high performance brakes, particularly for aircrafts. In the past, such brakes used carbon-carbon discs ${ }^{44}$ that have many excellent properties. They suffer, however, from such disadvantages as insufficient stability of friction caused by humidity and temperature and low oxidation resistance. Thus, carbon-SiC disks were introduced ${ }^{45-47}$ that represented a massive leap in the brakes' performance, particularly as it relates to fatigue resistance. The natural evolution of such brakes is to consider $\mathrm{SiC}-\mathrm{SiC}$ brakes, or perhaps ceramic composites containing $\mathrm{SiC}$. In addition, the problem is relevant to sliding of $\mathrm{SiC}$ fibers in the $\mathrm{SiC} /$ graphene/ $\mathrm{SiC}$ composite. ${ }^{48}$ Finally, the phenomenon is important to even fabrication of nanoporous membranes, ${ }^{39,40}$ since the nanoporous $\mathrm{SiC}$ layer is deposited on top of a mesoporous $\mathrm{SiC}$ layer (see below), and the static friction between the two layers during the deposition influences the structure and quality of the final product and, in particular, the interface between the mesoand nanoporous layers.

We have carried out extensive MD simulations to study the dry sliding friction between two $\mathrm{SiC}$ surfaces. Two types of surfaces have been considered. In one, we study kinetic friction at the interface between two amorphous $\mathrm{SiC}$ slabs, while in the second case, the $\mathrm{SiC}$ slabs have a crystalline structure. The goals are to understand the effect of the microstructure of the surface on the sliding friction and determine the dependence of the kinetic friction force on the sliding velocity. One expects, of course, higher friction between two amorphous surfaces than between the crystalline ones, but the magnitude of the difference, as well as any other factor contributing to the difference other than the roughness of the amorphous surface, is also important to understand.

The rest of this paper is organized as follows. In Sec. II, we describe the molecular model of the SiC slabs, as well as the force fields that we utilized in the MD simulations. Section III describes the development of the molecular model of the SiC-SiC interface, while Sec. IV explains the procedure for carrying out the MD simulations of dry sliding friction between the two SiC slabs. In Sec. V, we present and discuss the results and their implications. The paper is summarized in Sec. VI.

\section{MOLECULAR MODEL OF SiC AND THE FORCE FIELD}

The first step in the study is generating a molecular model of amorphous $\mathrm{SiC}$. We recently developed ${ }^{49-51}$ a model of amorphous $\mathrm{SiC}$ using the force field (FF) ReaxFF ${ }^{52}$ that has been developed for reactive environments. The goal in our previous work ${ }^{49-51}$ was to mimic the process by which a nanoporous membrane layer ${ }^{39,40}$ made of amorphous $\mathrm{SiC}$ is produced by the pyrolysis of a polymer precursor, allyl-hydridopolycarbosilane (AHPCS), which contains $\mathrm{Si}$. The primary product of the polymer's pyrolysis is amorphous $\mathrm{SiC}$ with covalent $\mathrm{Si}-\mathrm{C}$ bonds. Thus, we carried out ${ }^{49}$ quantummechanical calculations on model materials meant to capture important reaction steps and structures, in order to estimate the parameters of the ReaxFF. Then, a molecular model of the AHPCS was developed and ReaxFF was utilized to simulate its thermal degradation and decomposition as the system was heated up to $1200 \mathrm{~K}$ by the MD simulation. Analysis of the pyrolysis process and its products provided estimates of various quantities that were in good agreement with the experimental data. Finally, the system was cooled down to room temperature, with the final result being an amorphous $\mathrm{SiC}$ film. The computed properties of the resulting $\mathrm{SiC}$ film, such as its radial distribution function, X-ray diffraction pattern, and connectivity of its atoms, were in excellent agreement with the experimental data. In the present work, we use the molecular model of amorphous $\mathrm{SiC}$ that we had generated via the pyrolysis process and MD simulations.

The next step is to select the FF that describes the interactions between the two $\mathrm{SiC}$ surfaces. For this purpose, we also used ReaxFF. Both van der Waals and Coulombic interactions are included in ReaxFF. In addition, it should be kept in mind that ReaxFF eschews explicit bonds in favor of the bond order, which is useful for an environment in which bond breaking occurs regularly. The force applied to the system and the sliding friction heats up the region around the interface between the two $\mathrm{SiC}$ slabs, resulting 
in continuous breaking of the bonds there, which is why we opted to utilize ReaxFF that we developed ${ }^{49-51}$ previously.

\section{MODELS OF CONTACTING SiC SURFACES}

The unit cell for the amorphous $\mathrm{SiC}$ slabs was nonperiodic. Each slab was constructed with a size $2 a \times 2 a \times a$ that consisted of 2560 atoms, equally divided between $\mathrm{Si}$ and $\mathrm{C}$, where $a \approx 22 \AA$. The two slabs were identical and, thus, the system consisted of 5120 atoms. We also constructed two commensurate $(\beta) 3 \mathrm{C}-\mathrm{SiC}$ crystalline slabs, with each slab being a $8 b \times 10 b \times 4 b$ superlattice of the Si-C crystalline with 2560 atoms, where $b \approx 4.35 \AA$. The structure represented 16 planes of $\mathrm{Si}-\mathrm{C}$ planes stacked together.

Prior to the commencement of the main MD simulations of sliding friction, the energy of each individual slab was minimized. Then, MD simulation was used to minimize the energy between the surfaces prior to the commencement of the friction simulation, which produced minimal differences in the total potential energy of the system, implying correctness of the original structure of $\mathrm{SiC}$ that we had generated in our previous studies, ${ }^{49-51}$ and indicating that the slabs had indeed taken on their equilibrium structure. Next, the energy of the $\mathrm{SiC}-\mathrm{SiC}$ bilayer was minimized in order to prepare the system for thermalization. The atoms at the interface between the two slabs, for both amorphous and crystalline $\mathrm{SiC}$ slabs, were in "direct contact" with one another at the onset of the simulation, by which we mean that the separation distance between the $\mathrm{Si}$ and $\mathrm{C}$ atoms in the two slabs at their interface was at the minimum required to form a bond between them. We then thermalized the system in the NVT ensemble using the Lagevin thermostat and raised the temperature to $300 \mathrm{~K}$, and then equilibrated it in the NVE ensemble. This was done in a stepwise manner, with the system thermalized in increments of $50 \mathrm{~K}$, and then equilibrated after each incremental temperature rise. The duration of the thermalization and equilibration was 1200 ps.

\section{MOLECULAR DYNAMICS SIMULATION}

Figure 1 provides the details of the molecular system and the anchors to the amorphous bilayer. The MD simulations were carried out in the $(N V E)$ ensemble using the equilibrated interface. Sliding friction was generated by forcing the top slab to slide over the bottom one at a constant velocity $V$, which allowed us to determine the friction force for a given $V$. In order to simulate sliding friction at the interface, we divided the slabs into a series of layers, ${ }^{52-54}$ referring to them as the top layer (TL), top thermostat layer (TTL), bottom layer (BL), and bottom thermostat layer (BTL). The TTL and BTL were used to ensure that the upper and lower parts of the system remain at a constant temperature of $300 \mathrm{~K}$ throughout the duration of the MD simulations so that the generation of heat in the region around the interface can be clearly attributed to the sliding friction. This was done by rescaling the velocities of the atoms in the two layers at each time step. The atoms in the BL were fixed in all directions to ensure constant loading, while the atoms in the TL underwent two main events. First, in order to accurately mimic typical sliding experiments, it is necessary to apply normal loading to the system. This was accomplished by applying the force in the $-z$ direction to all the atoms in the TL. Two values of the normal forces were used that, at steady state, resulted in the system being exposed to pressures of

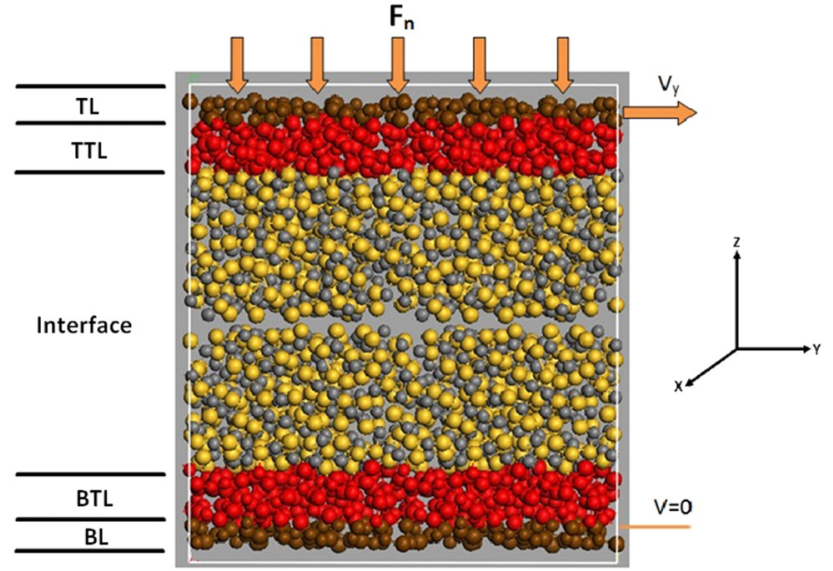

FIG. 1. Schematic of the system consisting of the two SiC slabs with the top layer (TL), top thermostat layer (TTL), the interface between the two slabs, the bottom thermostat layer (BTL), and the bottom layer (BL). $F_{n}$ is the normal force applied, while $V_{y}$ is the sliding velocity that pulls the top layer in the $y$ direction relative to the bottom slab.

approximately $155 \mathrm{MPa}$ and $310 \mathrm{MPa}$. Second, the atoms within the group TL were held constant at the prescribed velocity. The atoms directly at the interface may, however, have a different velocity at any given time. A constant velocity was applied in the $y$ direction to all the atoms in the TL, producing a fixed sliding velocity throughout the course of the MD simulations. Note that, strictly speaking, in the presence of friction, the relative velocity at the interface in the sticking regime (see below) should be zero, even though the relative motion of the TL and BL would be given by the prescribed velocity. In practice, however, there is always motion, albeit very slow, at the interface during the sticking regime, caused by shearing.

The same approach was also used with the crystalline bilayer. The simulated velocities were in the range $10-500 \mathrm{~m} / \mathrm{s}$, and each simulation run was carried out for $600-800$ ps that, after some preliminary simulations, had proven to be sufficiently long for our purpose. No melting was observed during any simulation at any velocity, as the melting temperature of $\mathrm{SiC}$ is $2730^{\circ} \mathrm{C}$. The time step in all the cases was $0.25 \mathrm{fs}$.

\section{RESULTS AND DISCUSSION}

We define the sliding distance $Y$ as the instantaneous difference between the $y$-positions of the centers of mass of the two slabs. For all the sliding velocities and distances, the temperature in the TTL and BTL fluctuated by only about $5 \%$, with the average values being around $303 \mathrm{~K}$, very close to the set temperature of 300 $\mathrm{K}$. The same type of variations of temperature with the sliding distance and velocity were obtained in the crystalline material.

Friction produces heat at the interface between the two slabs, however, which increases the temperature at and near the interface. The MD simulation confirmed this. Figure 2 presents the dynamic evolution of the temperature in the layer of the atoms in the top slab that are in direct contact (in the sense explained earlier) with those in 


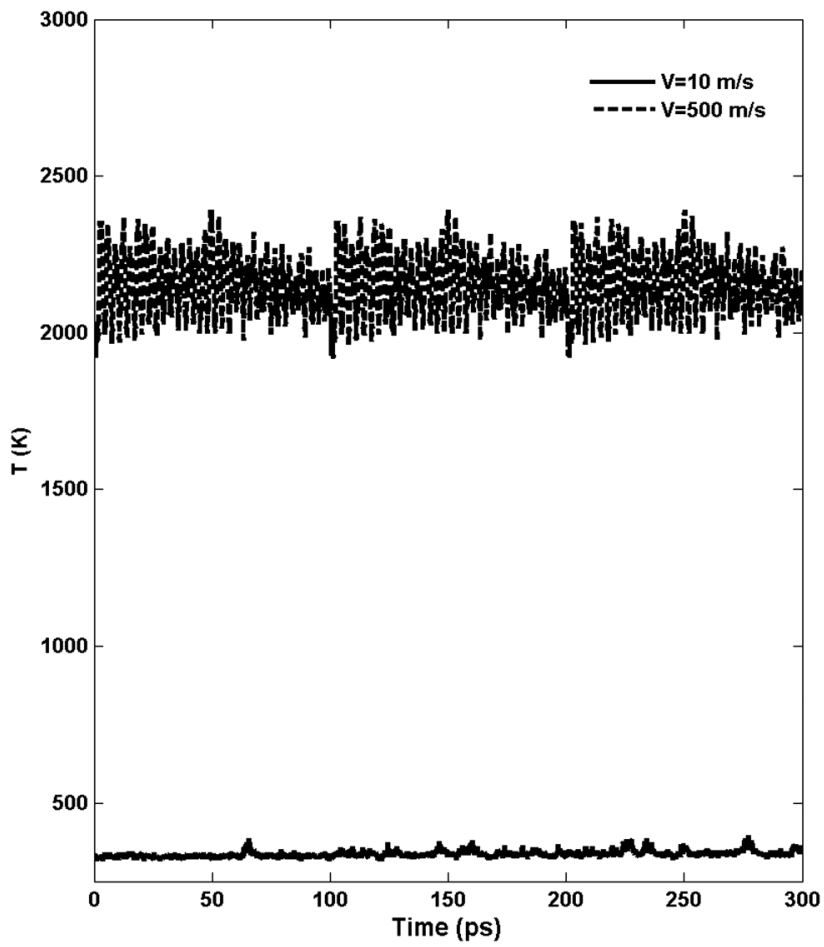

FIG. 2. Dynamic evolution of the temperature at the interface between two SiC slabs.

the bottom slab, which is where the maximum temperature develops. The results shown in Fig. 2 are for the lowest and highest sliding velocities $V$ that we simulated. As $V$ increases, so also does the heat production caused by friction, hence increasing the temperature there. The temperature between the interface and the TTL and BTL in which it is held fixed also increases. Figure 3 presents the dependence of the average interface temperature on the sliding velocity, indicating very large increase in the temperature at the interface.

Figure 4 presents the state of the two amorphous slabs and the interface between them, viewed in the $x$ direction (see Fig. 1 for the

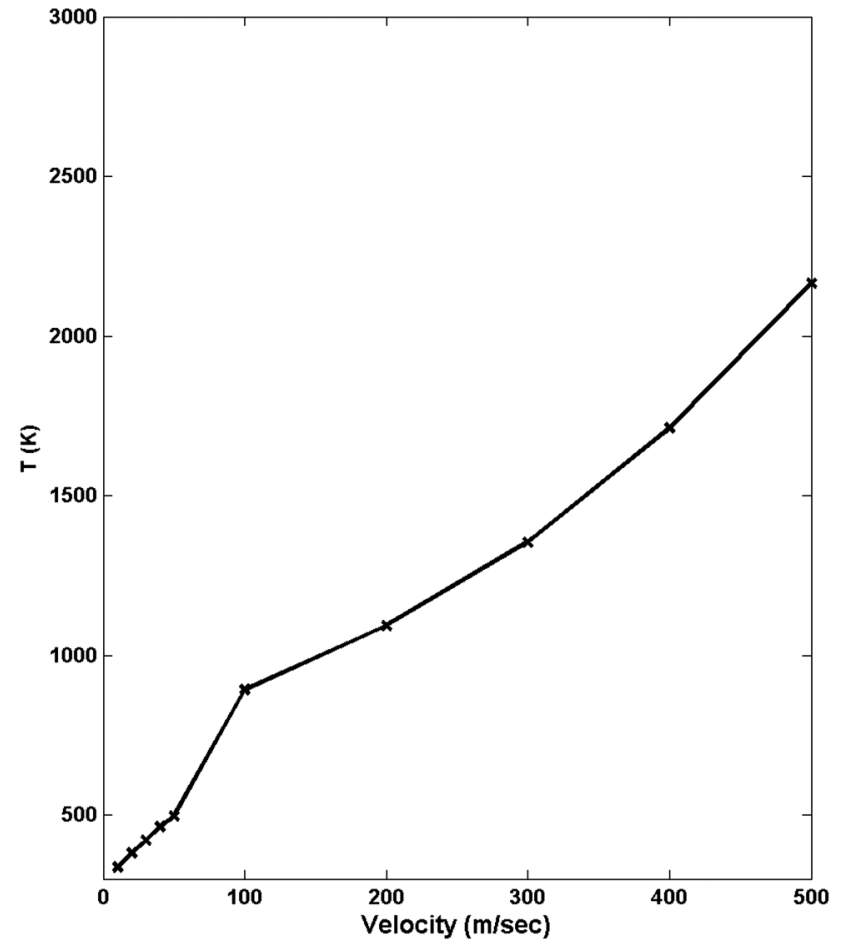

FIG. 3. Dependence of the average temperature at the interface between two amorphous slabs on the sliding velocity $V$.

coordinates system), as the sliding velocity $V$ increases. For low $V$, the two surfaces are in close contact. As $V$ increases, however, bond breaking due to heating, stretching, and deformation occurs at the interface, roughening the two surfaces, and generating a small gap or free volume at the interface whose extent increases with increasing sliding velocity. As we show below, this results in smaller frictional forces at the interface. The corresponding results for the crystalline slabs are shown in Fig. 5, indicating qualitative similarity with Fig. 4. The formation of the free volume is similar to the same type of phenomenon in metallic glasses. ${ }^{55-57}$ It is known, ${ }^{55,56}$ for

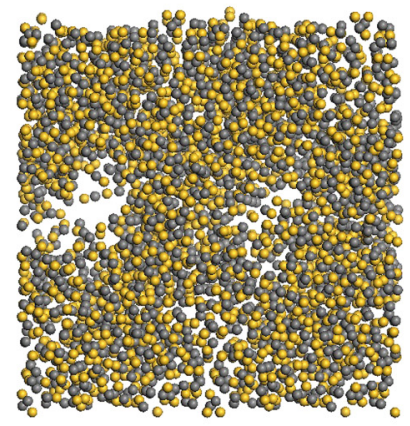

$10 \mathrm{~m} / \mathrm{sec}$

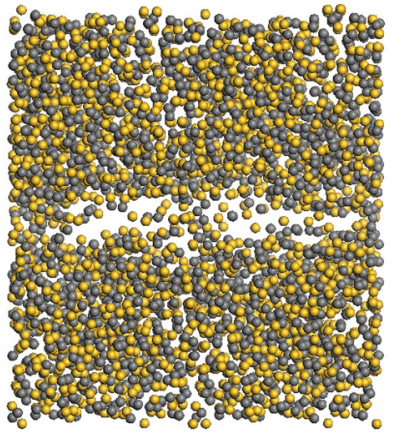

$100 \mathrm{~m} / \mathrm{sec}$

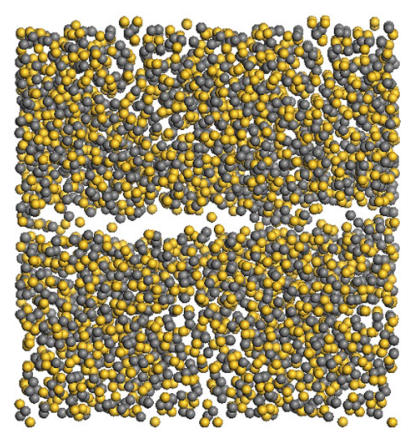

$500 \mathrm{~m} / \mathrm{sec}$
FIG. 4. Evolution of the excess free volume at the interface between two amorphous SiC slabs and its expansion as the sliding velocity increases. 


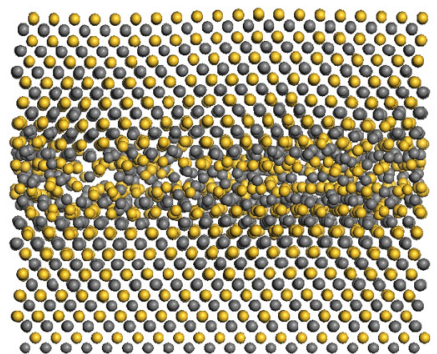

$10 \mathrm{~m} / \mathrm{sec}$

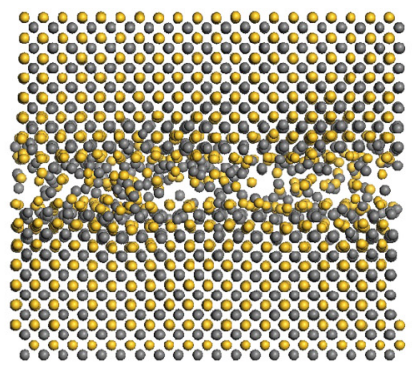

$100 \mathrm{~m} / \mathrm{sec}$

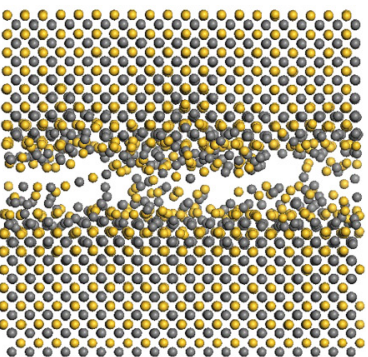

$500 \mathrm{~m} / \mathrm{sec}$
FIG. 5. Same as in Fig. 4, but in the system that consists of two crystalline slabs. example, that voids nucleate from the coalescence of excess free volume generated in shear bands during the deformation of some bulk metallic glass, such as $\mathrm{Zr}_{41.2} \mathrm{Ti}_{13.8} \mathrm{Cu}_{12.5} \mathrm{Ni}_{10.0} \mathrm{Be}_{22.5}$. The excess free volume in a shear band results in excess free energy relative to a relaxed glass with less free volume.

To better quantify and understand what Figs. 4 and 5 indicate, we computed the average number of atoms in thin parallelepipeds perpendicular to the $z$ direction during the entire simulation, for both the amorphous and crystalline systems. The results are presented in Fig. 6. As expected, far from the interface at $z=0$, the total number of atoms is constant. At $z=0$ and in the region around it, however, the number of atoms decreases due to bond breaking that occurs there. The depth of the "well" that represents the decrease in the number of the atoms depends on the sliding velocity $V$, and
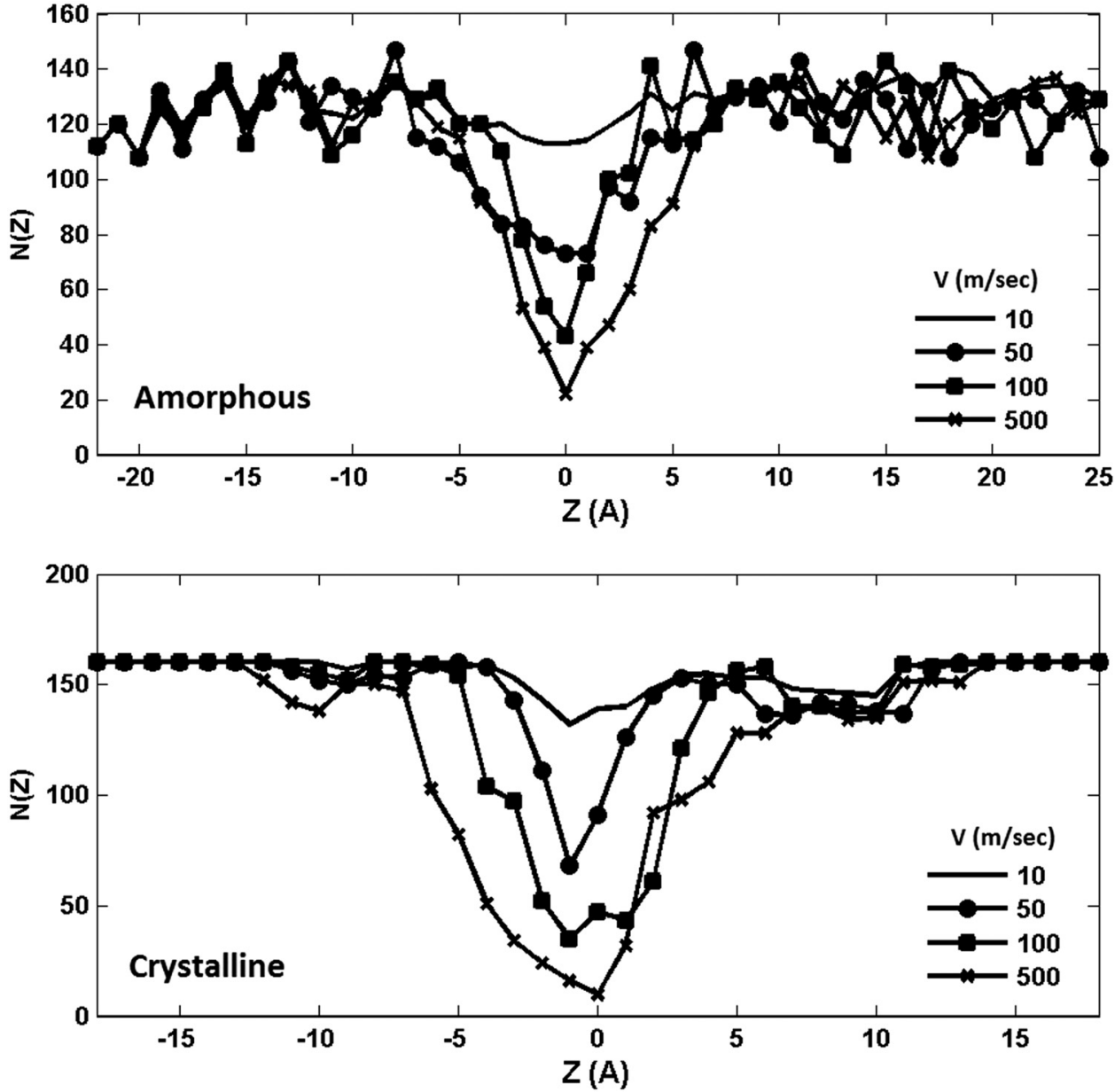

FIG. 6. Average number of atoms $N(z)$ in thin parallelepipeds perpendicular to the vertical axis $z$, and its dependence on the sliding velocity $V . z=0$ represents the interface, and the dip around it is due to the formation of excess free volume as a result of stretching and breaking the SiC bonds there. 
increases with increasing $V$. It is precisely due to this phenomenon that we used the force field ReaxFF in our MD simulation, even though other non-reactive FFs, such as the Tersoff potential, ${ }^{58,59}$ are available for $\mathrm{SiC}$ and $\mathrm{MD}$ simulations with them are faster.

Next, we examined the potential energy $U$ of the system, the sum of the energies of both slabs and the interface, which is an important characteristic of the system. The significance of $U$ is that its shape, for any given sliding velocity $V$, highlights important chemical and physical phenomena in nanoscale sliding that affect kinetic friction, as $U$ is greatly dependent upon the nature of the atoms at the interface and their spatial distribution. Due to the constant velocity $V$, the kinetic energy, aside from small fluctuations, remains essentially constant, hence enabling us to determine the kinetic friction force. Because the energy of the two slabs is constant as they slide relative to each other, the rate of change of $U$ along the sliding direction is linked directly with the instantaneous kinetic friction force, a fact that we exploit to study dynamic friction (see below). Because the velocity $V$ is fixed, the rate of change of sliding distance $Y$ with the time is also constant. We also point out that in all the cases, the potential energy of the amorphous system is significantly higher than its crystalline counterpart, reflecting a more unstable bilayer.

Figure 7 presents the dependence of $U$ on the sliding distance $Y$ for the lowest and highest sliding velocities in the amorphous system. The atomistically rough surfaces of the two slabs give rise to a complex relationship between $U$ and $Y$. At low velocities, the motion is intermittent, with $U$ varying with the sliding distance periodically, which is typical of stick-slip motion, stemming from the rough nature of the interface that contributes to the variations in the friction intensity. Note that such a periodicity in the stickslip motion has been observed in experiments ${ }^{32}$ by using an atomic force microscope. The maxima in $U$ correspond to the slip forces, whereas the minima represent the stick state. At much higher velocities, however, the motion is fast and the system does not have enough time to relax. Thus, the variations of $U$ with the sliding distance is stronger, although they still appear to be quasi-periodic. Note that the potential energy grows roughly quadratically with the distance in the stick regime
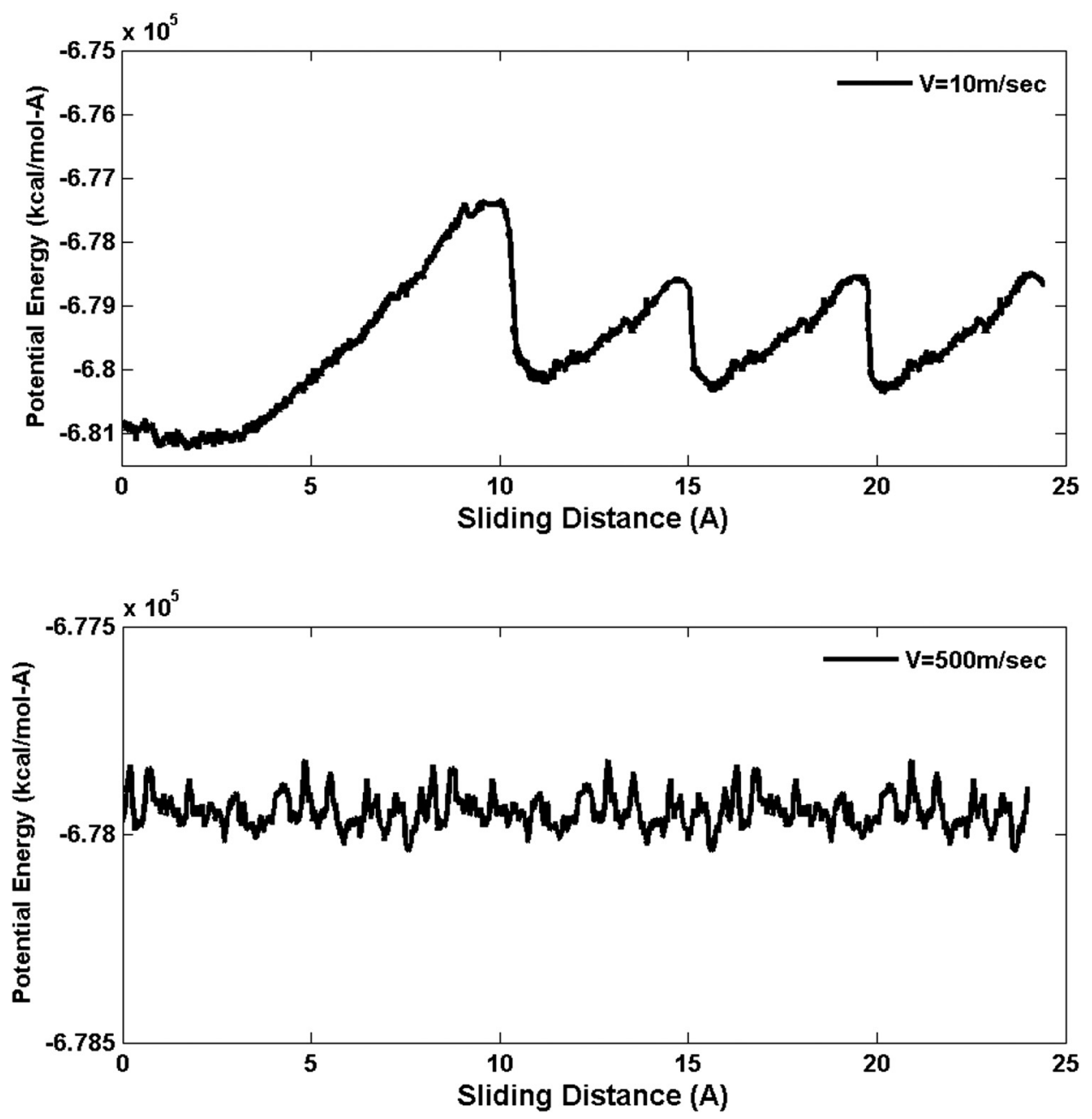

FIG. 7. Dependence of the potential energy of the amorphous system on the sliding velocity $V$ and sliding distance. 
We define the friction force $F_{y}$ per contact area in terms of the potential energy through the relation

$$
F_{y}=-\frac{1}{A} \frac{d U(Y)}{d Y}
$$

where $A$ is the contact area. Alternatively, one may compute the friction force $F_{y}$ directly using MD calculation's output, as it represents the total lateral atomic force on the upper slab, divided by the contact area at each MD time step. In other words, $F_{y}$ is simply the sum of all the atomic forces along the $y$ direction. But, only the lateral forces at the interface contribute to $F_{y}$, as the internal forces cancel each other in the sum. This is the approach we took in this paper. $F_{y}$ is an important physical quantity because it represents the intensity of the friction force. Equation (1) and Fig. 7 do, however, provide a qualitative picture of what one may expect for $F_{y}$ as a function of the sliding distance $Y$ : at low sliding velocity $F_{y}$ should vary periodically with $Y$, but as $V$ increases, the system does not have enough time to relax, and the periodicity is to some extent distorted.
Suppose that the static potential energy corresponding to $V=0$ is $U_{0}(Y)$, implying that $U_{0}(Y)$ represents only the potential energy due to interfacial bonding or adhesion. Thus, the difference $U(Y)-U_{0}(Y)$ is purely due to the sliding velocity. The force needed to overcome the energy barriers in $U_{0}(Y)$ is $d U_{0}(Y) / d Y$, which is due to the change in the interfacial energy and represents a lower bound on $F_{y}$, the instantaneous kinetic frictional force during sliding. Figure 8 presents the directly computed $F_{y}$ [i.e., not through Eq. (1)] as a function of the sliding distance $Y$ and the sliding velocity $V$. It is clear that our expectations, described earlier based on $d U(Y) / d Y$, for the differences between $F_{y}$ at low and high velocities are precisely manifested by the results. The results shown in Fig. 8 were computed by applying a normal force $F_{n}$ of $2.85 \mathrm{nN}$ to the system. Qualitatively, similar results were obtained when the magnitude of $F_{n}$ was higher and, thus, the results are not shown.

At low sliding velocities, the variations in $F_{y}$ are periodic, representing stick-slip motion. During the stick state, the force $d U / d Y$ causes the slab to store strain energy through shearing, which is then released during slip. The minima in $F_{y}$ correspond to the slip
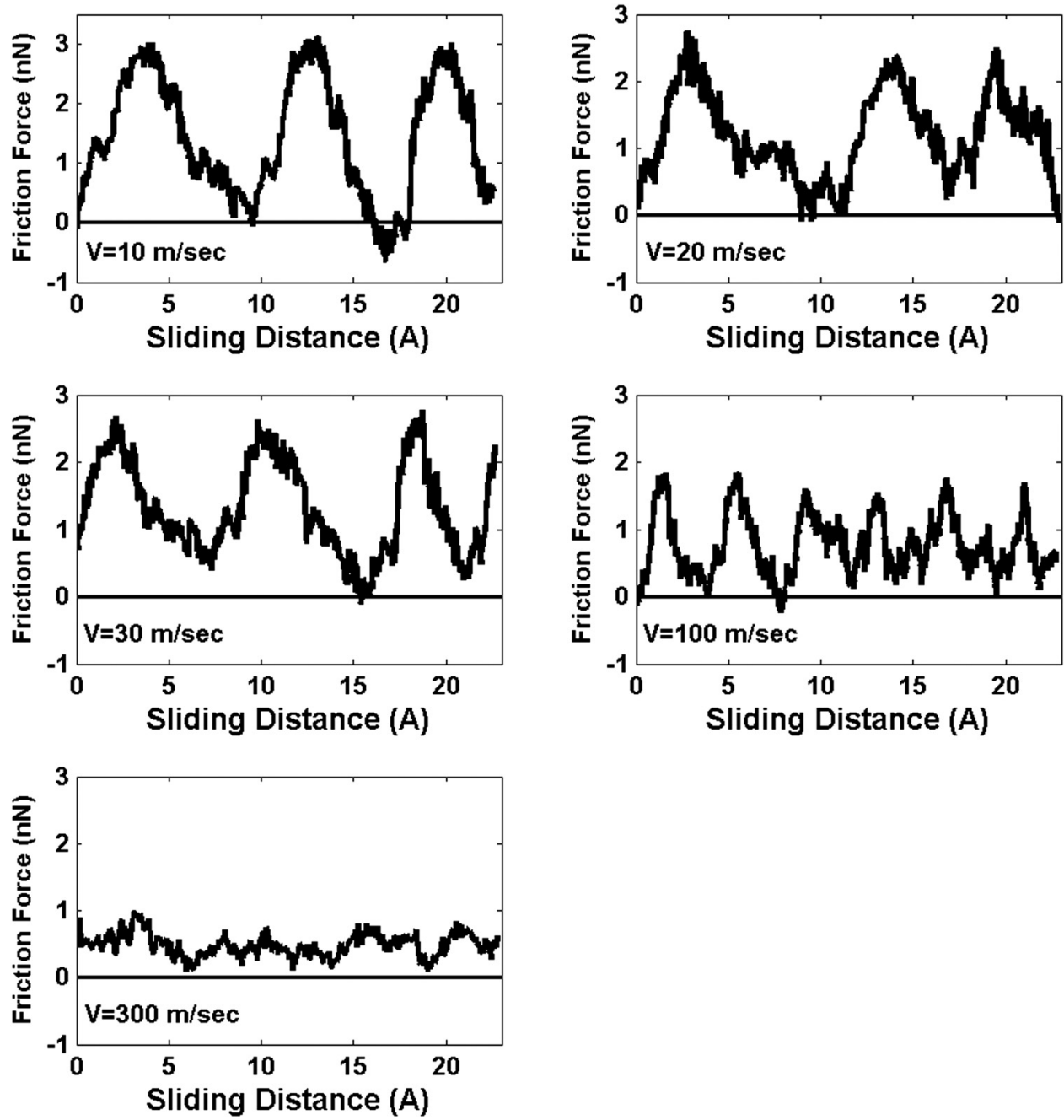

FIG. 8. Dependence of the friction force on the sliding distance and velocity $V$ in the amorphous system. 
forces, whereas the approach to the maxima of $F_{y}$ represents the stick motion, and they both are larger than those for $V=0$, i.e., the aforementioned $d U_{0}(Y) / d Y$. Note that the difference between the minimum and maximum values of $F_{y}$ at low velocities is relatively significant, whereas the same difference is small at high velocities. Once again, similar to the potential energy $U(Y)$, the force $F_{y}$ at high velocities varies with smaller amplitudes and, due to bond breaking at the interface, its magnitude also decreases.

Figure 9 presents the same results as in Fig. 8, but for the crystalline slabs. We first note that the range of friction intensities for amorphous $\mathrm{SiC}$ is significantly greater than that of the crystalline slabs. Due to the crystalline structure, the periodic variations of the friction force, a characteristic of the stick-slip motion, are even more evident than in the amorphous bilayer. Similar to the amorphous case, the amplitude of the variations diminishes with increasing sliding velocity, and the periodic structure is not as well-defined. As the sliding velocity increases, however, the distinction between the amorphous and crystalline interfaces is lost due to the bond breaking and roughening of the interface and, therefore, at such velocities, the friction forces in both materials are close to each other.

Higher friction forces are associated with increased probability of reaching fatigue stresses, usually defined as the highest stress that a material can withstand for a given number of cycles without breaking, which destabilize the material, causing loss of structural integrity. At high velocities, $F_{y}$ in the crystalline slabs still exhibits peaks and valleys, but the stick-slip behavior discussed earlier is less clear due to the fluctuations from the thermal phonons in crystalline materials, and the fact that there is less time for the system to relax. In addition, at high velocities, thermal phonons are also responsible for damping of slab motion, hence slowing down the increase in $F_{y}$ with velocity $V$. Indeed, if we write $U(Y)=U_{0}(Y)+U_{e}(Y, V)+U_{p}(Y, V)$, where $U_{e}$ is the elastic strain energy and $U_{p}$ is the elastic energy due to phonons, at low $V$, the first two terms of the equation are dominant, whereas at higher velocities, say $V>25 \mathrm{~m} / \mathrm{s}$, it is only the last term that contributes significantly to $U(Y)$ and $F_{y}$, hence explaining the decrease in the magnitude of $F_{y}$.
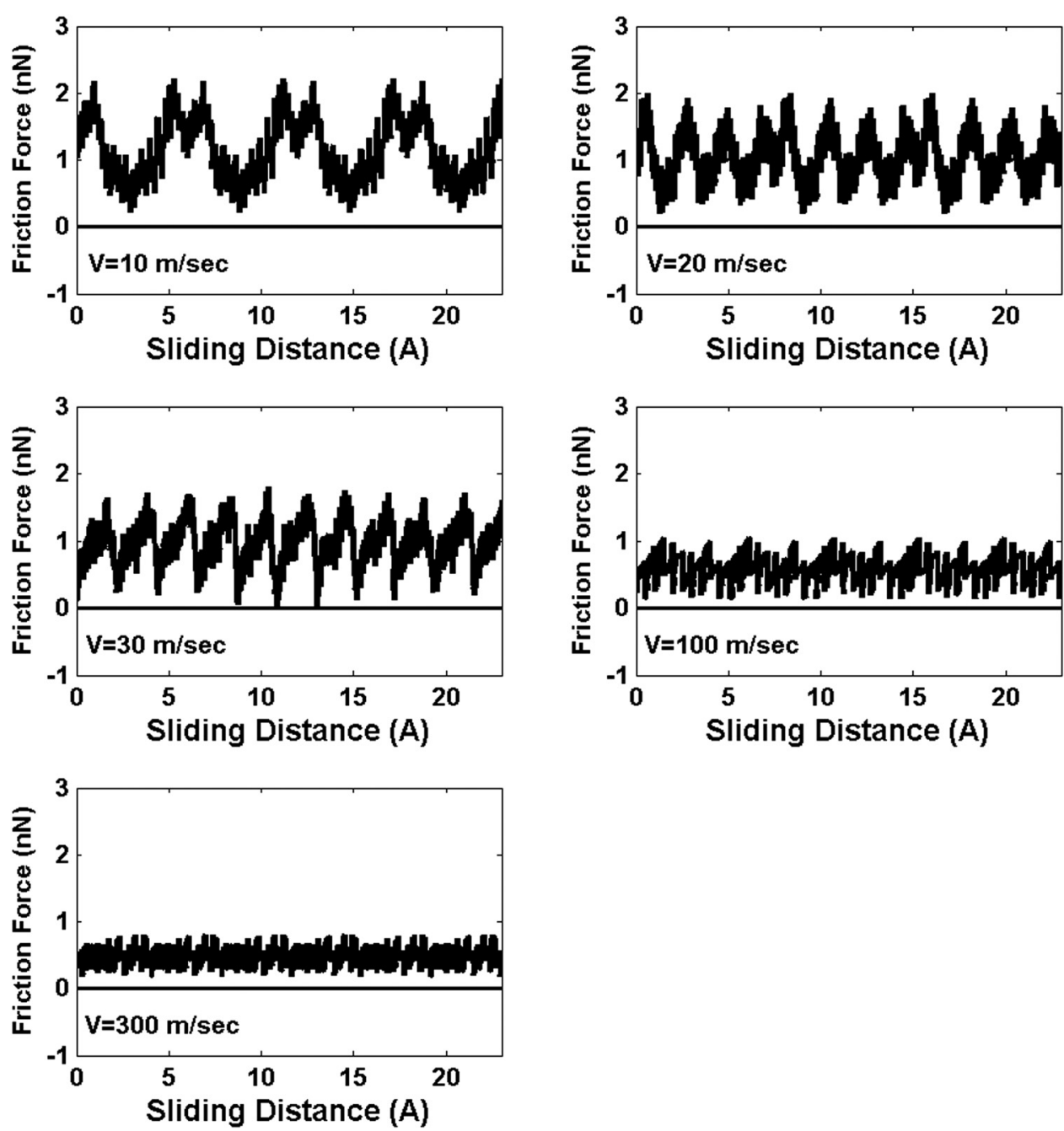

FIG. 9. Same as in Fig. 8, but for friction force between two crystalline slabs. 
The decrease in the friction force with an increasing sliding velocity has been seen in experimental studies. ${ }^{53,54}$ The reason for this is twofold. One, as already pointed out, is that there is less time for adhesion to take place at the interface at high sliding velocities and, therefore, shorter times for the stick-slip motion. The second reason is that even in the crystalline system, the interface becomes increasingly roughened and more similar to an amorphous material, and a low-density region forms near the interface as the sliding velocity increases. This is then very much similar to the aforementioned concept of excess free volume; see Fig. 6 .

The extent of shearing of the system is quantified by position $Y_{0}$ of the center of mass of the outermost layer, as well as that of the interface layer, $Y_{i}$. Thus, let, $\Delta Y=Y_{0}-Y_{i}$, which is proportional to the shear strain. In the absence of shearing, $\Delta Y$ will not change. The regions in Figs. 8 and 9 in which $F_{y}>0$ represent those for which $\Delta Y>0$, i.e., forward or positive shearing of the top slab. The sliding distance from a maximum of $\Delta Y$ to its minimum represents the actual slip distance, which is much smaller than the distance through which $\Delta Y$ increases during the stick motion. This is particularly true at high velocities.

We also calculated the time-average of the instantaneous intensity of the frictional force, $\left\langle F_{y}\right\rangle$, over long sliding distances. The kinetic frictional force intensity $F_{k}$ is defined by

$$
F_{k}=\left\langle F_{y}\right\rangle
$$

which is valid for any sliding velocity. Of course, if the potential $U(Y)$ were exactly symmetric for all $Y$, then $F_{k}=0$, because the sticking and slipping forces would be exactly equal but with opposite signs. But, as the results presented earlier indicated, $U(Y)$ is not exactly periodic or symmetric and, therefore, its slope that yields $F_{y}$ varies, i.e., we expect a nonzero $F_{k}$.

Figure 10 presents the plot of $F_{k}$ vs $V$, and it is clear that the dependence of friction force on the sliding velocity is nonlinear. In fact, Fig. 10 indicates that the kinetic friction force $F_{k}$ roughly follows, $F_{k} \propto \ln V$, which had been reported for friction on the $\mathrm{NaCl}$ (100) surfaces $^{18}$ at low velocities. According to Fig. 10, up to about $V \approx 35 \mathrm{~m} / \mathrm{s}, F_{k}$ depends on $V$ as $\ln V$. There seems to be a transition for $35<V<55 \mathrm{~m} / \mathrm{s}$, beyond which the logarithmic dependence is roughly followed again. Figure 10 also demonstrates the difference between sliding frictions in the two types of materials that we study, particularly at low sliding velocities. According to Fig. 10, for $V=0$, one has $F_{s}=\left\langle d U_{0}(Y) / d Y\right\rangle \approx 1.8$ and $1.4 \mathrm{nN}$ for, respectively, the amorphous and crystalline surfaces. Coulomb's law asserts that $F_{k}$ is independent of the sliding velocity $V$. The important implication of Fig. 10 is, however, that $F_{k}$ does depend on $V$. That is, at the nanoscale that we have studied, Coulomb's law is not satisfied.

For macroscopic surfaces, the friction coefficient $\mu$ is defined by, $\mu=F_{k} / F_{n}$, where $F_{n}$ is the normal force applied on one of the two slabs. The discussions in the literature on the sliding friction between two nanoscale surfaces may be divided into two groups. In one group are ${ }^{29,53}$ those that describe the phenomenon in terms of the relations between the friction force $F_{y}$ and its average $F_{k}$ and the sliding distance and velocity, as we described earlier, in order to understand whether macroscopic friction laws are still applicable at

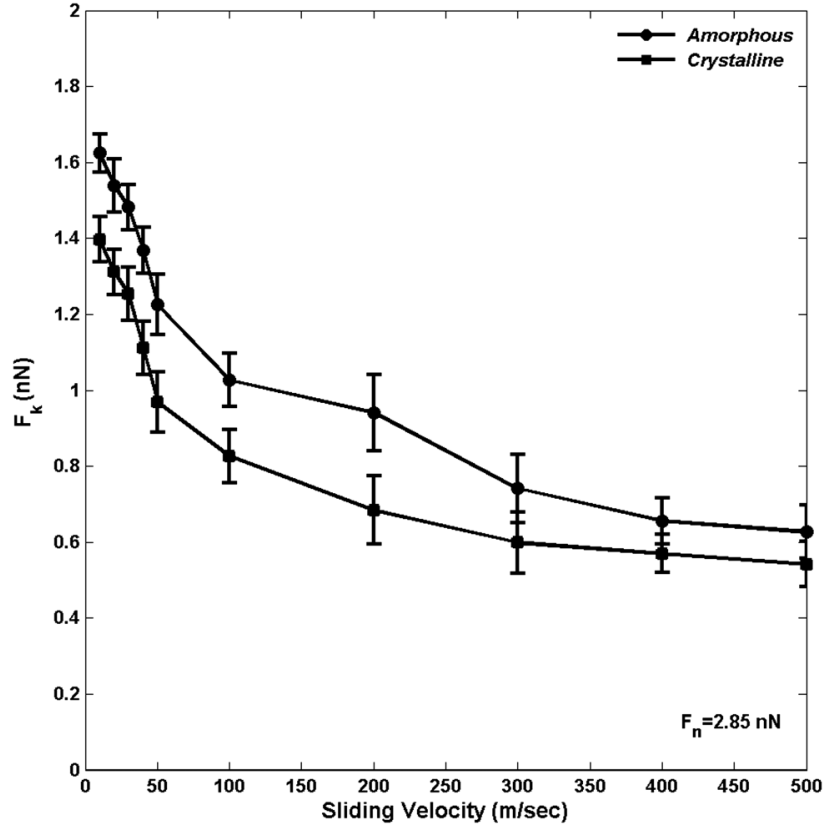

FIG. 10. Dependence of the kinetic friction force $F_{k}$ on the sliding velocity $V$. For $V \leq 35 \mathrm{~m} / \mathrm{s}$, dependence of $F_{k}$ on $V$ is perfectly logarithmic, while after a transition region, the same type of dependence appears to be roughly followed.

the nanoscale. In the second group are those ${ }^{60}$ that use the aforementioned definition of $\mu$ at the nanoscale in order to extract a dynamic friction coefficient. We also point out that using the Prandtl-Tomlinson model of nanotribology ${ }^{61,62}$ one can show straightforwardly that the static value of $F_{k}$, i.e., its value in the limit $V=0$, varies linearly with $F_{n}$ so that the static friction coefficient $\mu_{s}$ can be estimated as a result of varying $F_{n}$.

If we were to follow the second group, then, we could obtain an estimate of the static friction coefficient $\mu_{s}$ between the two $\mathrm{SiC}$ surfaces by extrapolating the results to the limit $V=0$. In that case, we find that for the amorphous bilayer, $\mu_{s} \approx 0.6$, whereas for the crystalline surfaces, $\mu_{s} \approx 0.52$. Experimental values are in the range ${ }^{63,64} 0.45-0.5$.

\section{SUMMARY}

Due to its numerous important applications, it is of fundamental interest to study the dependence of kinetic friction between two $\mathrm{SiC}$ slabs on the sliding velocity. In addition, since $\mathrm{SiC}$ exists both as an amorphous material and in the crystalline form, the effect of surface roughness on the kinetic friction is also of importance. This paper reported the results of an extensive study by MD simulation of sliding friction between the two types of SiC surfaces. With increasing sliding velocity, bond breaking occurs at the interface between the two surfaces, leading to their roughening and formation of excess free volume. The roughening and excess free volume reduce the kinetic friction force, leading to a reduction in the difference between kinetic friction in the amorphous and 
crystalline surfaces. The kinetic friction force appears to depend logarithmically on the sliding velocity.

\section{SUPPLEMENTARY MATERIAL}

In the supplementary material, we present the input file used to carry out the molecular dynamics calculations, containing the steps for minimization, thermalization, and simulation of friction dynamics. In addition, the optimized geometry for both amorphous and crystalline $\mathrm{SiC}$ is presented.

\section{ACKNOWLEDGMENTS}

The computations were carried out using the facilities of the University of Southern California High Performance Computing Center.

\section{REFERENCES}

'B. Feeny, A. Guran, N. Hinrichs, and K. Popp, Appl. Mech. Rev. 51, 321 (1998). ${ }^{2}$ B. N. J. Persson, Sliding Friction: Physical Principles and Applications (Springer, New York, 2000).

${ }^{3}$ Principles of Tribology, edited by J. Halling (MacMillan, London, 1978).

${ }^{4}$ M. C. Shaw, A. Ber, and P. A. Mamin, J. Basic Eng. 82, 342 (1960).

${ }^{5}$ J. R. Rice and A. L. Ruina, J. Appl. Mech. 50, 343 (1983).

${ }^{6}$ B. Jacobson, Tribology Int. 36, 781 (2003).

${ }^{7}$ D. Dowson, History of Tribology, 2nd ed. (American Society of Mechanical Engineers, New York, 1999).

${ }^{8}$ B. Bhushan, J. N. Israelachvili, and U. Landman, Nature 374, 607 (1995).

${ }^{9}$ E. D. Smith, M. O. Robbins, and M. Cieplak, Phys. Rev. B 54, 8252 (1996).

${ }^{10}$ W. F. Brace and J. D. Byerleev, Science 153, 990 (1966).

${ }^{11}$ C. H. Scholz, Nature 391, 37 (1998).

${ }^{12}$ S. D. Mesarovic, J. Mech. Phys. Solids 43, 671 (1995).

${ }^{13}$ M. A. Heckel and I. D. Abrahams, J. Sound Vib. 229, 669 (2000).

${ }^{14}$ L. G. Hector, Jr and B. Schmid, Wear 215, 257 (1998).

15J. Krim, D. H. Solina, and R. Chiarello, Phys. Rev. Lett. 66, 181 (1991).

${ }^{16} \mathrm{O}$. Zwörner, H. Hölscher, U. D. Schwarz, and R. Wiesendanger, Appl. Phys. A 66, S263 (1998)

${ }^{17}$ E. Gnecco, R. Bennewitz, T. Gyalog, Ch. Loppacher, M. Bammerlin, E. Meyer, and H. J. Göntherodt, Phys. Rev. Lett. 84, 1172 (2000).

${ }^{18}$ U. Bockelmann, B. Essevaz-Roulet, and F. Heslot, Phys. Rev. Lett. 79, 4489 (1997).

19. A. Harrison, C. T. White, R. J. Colton, and D. W. Brenner, Phys. Rev. B 46, 9700 (1992).

20 J. N. Glosli and G. M. McClelland, Phys. Rev. Lett. 70, 1960 (1993).

${ }^{21}$ L. Zhang and H. Tanaka, Wear 211, 44 (1997).

22 J. Shimizu, H. Eda, M. Toritsune, and E. Ohmura, Nanotechnology 9, 118 (1998).

${ }^{23}$ G. He, M. H. Müser, and M. O. Robbins, Science 284, 1650 (1999).

${ }^{24}$ M. H. Müser and M. O. Robbins, Phys. Rev. B 61, 2335 (2000).

${ }^{25}$ B. Li, P. C. Clapp, J. A. Rifkin, and X. M. Zhang, J. Appl. Phys. 190, 3090 (2001).

${ }^{26}$ M. H. Müser, Phys. Rev. Lett. 89, 224301 (2002).

27. Zhang and J. B. Sokoloff, Phys. Rev. E 71, 066125 (2005).

${ }^{28}$ E. S. Torres, S. Goncalves, C. Scherer, and M. Kiwi, Phys. Rev. B 73, 035434 (2005).
${ }^{29}$ Q. Zhang, Y. Qi, L. G. Hector, Jr., T. Cagin, W. A. Goddard III, Phys. Rev. B 72, 045406 (2005).

${ }^{30}$ W. K. Kim and M. L. Falk, Model. Simul. Mater. Sci. Eng. 18, 034003 (2010).

${ }^{31}$ X. J. Yang, Appl. Mech. Mater. 345, 167 (2013).

${ }^{32}$ S. Kawai, A. Benassi, E. Gnecco, H. Söde, R. Pawlak, X. Feng, K. Müllen, D. Passerone, C. A. Pignedoli, P. Ruffieux, R. Fasel, and E. Meyer, Science 351, 957 (2016).

${ }^{33}$ Y. Takeda, K. Nakamura, K. Maeda, and Y. Matsushita, J. Am. Ceram. Soc. C-70, 266 (1987).

${ }^{34}$ K. Schulz and M. Durst, Filtr. Sep. 31, 25 (1994).

${ }^{35}$ A. J. Rosenbloom, D. M. Sipe, Y. Shishkin, Y. Ke, R. P. Devaty, and W. J. Choyke, Biomed. Microdev. 6, 261 (2004).

${ }^{36} \mathrm{Z}$. Li, K. Kusakabe, and S. Morooka, J. Membr. Sci. 118, 159 (1996).

${ }^{37}$ C. A. Zorman, A. J. Fleischman, A. S. Dewa, M. Mehregany, C. Jacob, S. Nishino, and P. Pirouz, J. Appl. Phys. 78, 5136 (1995).

${ }^{38}$ S. H. Kenawy and W. M. N. Nour, J. Mater. Sci. 40, 3789 (2005).

${ }^{39}$ B. Elyassi, M. Sahimi, and T. T. Tsotsis, J. Membr. Sci. 288, 290 (2007).

${ }^{40}$ B. Elyassi, M. Sahimi, and T. T. Tsotsis, J. Membr. Sci. 316, 73 (2008).

${ }^{41}$ N. Keller, C. Pham-Huu, G. Ehret, V. Keller, and M. J. Ledoux, Carbon 41, 2131 (2003).

${ }^{42}$ S. H. Barghi, T. T. Tsotsis, and M. Sahimi, Int. J. Hydrogen Energy 39, 21107 (2014).

${ }^{43}$ S. H. Barghi, T. T. Tsotsis, and M. Sahimi, Int. J. Hydrogen Energy 41, 369 (2016).

${ }^{44}$ W. Krenkel and T. Henke, Key Eng. Mater. 164-165, 421 (1999).

${ }^{45}$ W. Krenkel, B. Heidenreich, and R. Renz, Adv. Eng. Mater. 4, 427 (2002).

${ }^{46}$ S. Fan, L. Zhang, Y. Xu, L. Cheng, J. Lou, J. Zhang, and L. Yu, Compos. Sci. Technol. 67, 2390 (2007)

${ }^{47}$ S. Fan, L. Zhang, Y. Xu, L. Cheng, G. Tian, S. Ke, F. Xu, and H. Liu, Compos. Sci. Technol. 68, 3002 (2008)

${ }^{48}$ J. B. Wallace, D. Chen, and L. Shao, Mater. Res. Lett. 4, 55 (2016).

${ }^{49}$ S. Naserifar, L. Liu, W. A. Goddard III, T. T. Tsotsis, and M. Sahimi, J. Phys. Chem. C 117, 3308 (2013).

${ }^{50}$ S. Naserifar, W. A. Goddard III, L. Liu, T. T. Tsotsis, and M. Sahimi, J. Phys. Chem. C 117, 3320 (2013).

${ }^{51}$ S. Naserifar, W. A. Goddard III, T. T. Tsotsis, and M. Sahimi, J. Chem. Phys. 142, 174703 (2015).

${ }^{52}$ A. C. T. van Duin, S. Dasgupta, F. Lorant, and W. A. Goddard, J. Phys. Chem. A 105, 9396 (2001)

${ }^{53}$ A. Li, Y. Liu, and I. Szlufarska, Tribol. Lett. 56, 481 (2014).

${ }^{54}$ C. Hu, M. Bai, J. Lv, Z. Kou, and X. Li, Tribol. Int. 90, 297 (2015).

${ }^{55}$ C. Nagel, K. Rätzke, E. Schmidtke, J. Wolff, U. Geyer, and F. Faupel, Phys. Rev. B 57, 10224 (1998).

${ }^{56}$ W. J. Wright, T. C. Hufnagel, and W. D. Nix, J. Appl. Phys. 93, 1432 (2003).

${ }^{57}$ A. R. Yavari, A. Le Moulec, A. Inoue, N. Nishiyama, N. Lupu, E. Matsubara, W. J. Botta, G. Vaughan, M. Di Michiel, and A. Kvick, Acta Mater. 53, 1611 (2005).

${ }^{\mathbf{5 8}}$ J. Tersoff, Phys. Rev. Lett. 61, 2879 (1988).

59. Tersoff, Phys. Rev. B 37, 6991 (1988).

${ }^{60}$ Y. Wang, J. Xu, J. Zhang, Q. Chen, Y. Ootani, Y. Higuchi, N. Ozawa, J. M. Martin, K. Adachi, and M. Kubo, Carbon 133, 350 (2018).

${ }^{61}$ L. Prandtl, Z. Angew. Math. Mech. 8, 85 (1928).

${ }^{62}$ G. A. Tomlinson, Philos. Mag. 7, 905 (1929).

${ }^{63}$ X. Zhao, Y. Liu, Q. Wen, and Y. Wang, Friction 2, 58 (2014).

${ }^{64}$ S. Lafon-Placette, K. Delbé, J. Denape, and M. Ferrato, J. Eur. Ceram. Soc. 35, 1147 (2015). 